\title{
Valoração econômica de bens e serviços ecossistêmicos no Parque Nacional da Serra da Capivara: uma abordagem baseada no Método do Custo de Viagem
}

\author{
Economic valuation of ecosystem goods and services in the Serra da \\ Capivara National Park: an approach based on the Travel Cost Method
}

\author{
Elinne Nastasha Carvalho Sousa Val1 \\ Alain Hernández Santoyo ${ }^{2}$ \\ Deive Ciro de Oliveira ${ }^{3}$ \\ Weimar Freire da Rocha $\mathrm{Jr}^{4}$
}

\begin{abstract}
Palavras-chave:
Economia Ambiental

Método de Preferências Reveladas

Valor de Uso Recreativo

Excedente do Consumidor

Resumo

O seguinte artigo objetivou estimar o valor de uso recreativo dos bens e serviços ecossistêmicos do Parque Nacional da Serra da Capivara, localizado no Sul do estado do Piauí, região Nordeste brasileira, com uma notável atração de um turismo baseado na natureza, história e cultura. Utilizou-se o Método do Custo de Viagem por meio da aplicação de questionário online para os turistas que realizaram visitas ao local, cujas informações foram coletadas durante o mês de junho de 2019. Constatou-se que o perfil dos visitantes inclui, maiormente, pessoas com um grau de escolarização e nível de renda mais altos, os quais avaliaram com maior nota a observação de paisagem nos mirantes, visitas ao Museu da Natureza e os sítios arqueológicos. Como consenso, quase a totalidade dos usuários e dos não usuários reconhece a relevância deste espaço natural e estão dispostos a pagar por uma taxa de ingresso, a qual ajudaria na conservação e sustentação do lugar. O valor de uso recreativo do Parque Nacional da Serra da Capivara, estimado a partir do Método de Custo da Viagem, foi de $\mathrm{R} \$ 7,1$ milhões por ano, resultado que mesmo sendo impressionante, ainda pode estar subestimado, pois o valor de uso potencial estimado alcançou $\mathrm{R} \$ 2,1$ bilhões, confirmando a importância dos investimentos em infraestrutura para o receptivo, assim como em mecanismos de proteção ambiental que propiciem o conhecimento e manutenção da integridade ecológica do Parque e da atividade econômica de turismo que ele sustenta.
\end{abstract}

${ }^{1}$ Universidade Federal de Alfenas - UNIFAL/MG, Programa de Pós-Graduação em Economia (PPGEconomia), Campus Varginha, Minas Gerais.elinneval@gmail.com.

2 Universidade Federal de Alfenas - UNIFAL/MG, Programa de Pós-Graduação em Economia (PPGEconomia), Campus Varginha, Minas Gerais. santoyocuba@gmail.com.

${ }^{3}$ Universidade Federal de Alfenas - UNIFAL/MG, Instituto de Ciências Sociais Aplicadas (ICSA), Campus

Varginha, Minas Gerais. deive.oliveira@unifal-mg.edu.br.

${ }^{4}$ Universidade Estadual do Oeste do Paraná - UNIOESTE, Programa de Pós-Graduação em Desenvolvimento Regional e Agronegócio (PGDRA), Campus Toledo, Paraná. wrochajr2000@gmail.com. 


$\begin{array}{ll}\text { Keywords } & \text { Abstract } \\ \text { Environmental economics } & \text { This article aimed to estimate the recreational use value of } \\ \text { Revealed Preferences Method } & \text { ecosystem goods and services in the Serra da Capivara National } \\ \text { Recreational Use Value } & \text { Park, located in the south of the state of Piauí, in the Northeast } \\ \text { onsumer Surplus } & \text { history and culture. The Travel Cost Method was used through } \\ \text { he application of an online questionnaire for tourists who made } & \text { visits to the location, whose information was collected during } \\ \text { the month of June 2019. It was found that the profile of visitors } \\ \text { includes, mostly, people with a higher level of schooling and } \\ \text { income level, which rated the observation of the landscape in } \\ \text { the viewpoints, visits to the Museum of Nature and the } \\ \text { archaeological sites more highly. As a consensus, almost all } \\ \text { users and non-users recognize the relevance of this natural } \\ \text { space and are willing to pay an entrance fee, which would help } \\ \text { in its conservation and support. The recreational use value of } \\ \text { the Serra da Capivara National Park estimated from the Cost } \\ \text { of Travel Method was R } \$ \text { 7.1 million per year, a result that } \\ \text { although impressive, may still be underestimated, since the } \\ \text { estimated potential use value reached R } \$ 2.1 \text { billion, confirming } \\ \text { the importance of investments in infrastructure for the } \\ \text { reception of visitors, as well as in environmental protection } \\ \text { mechanisms that provide knowledge and maintenance of the } \\ \text { ecological integrity of the Park and the economic tourism } \\ \text { activity that it sustains. }\end{array}$

\section{INTRODUÇÃO}

Desde o final do século XIX, há registros da existência de áreas naturais protegidas com o objetivo de preservar e evitar a degradação ambiental. No entanto, além de ser essencial proteger o espaço biofísico, é necessário envolver a sociedade neste propósito, por meio de atividades culturais, educacionais, científicas, socioeconômicas e recreativas, as quais, de acordo com Mesquita et al (2015) têm finalidades comerciais capazes de gerar receitas financeiras e desenvolvimento social e econômico em diversos locais do mundo. Dentre as múltiplas atividades mencionadas, as recreativas têm experimentado uma expansão relevante na demanda das pessoas para fazer uso de um ambiente de alta qualidade que ofereça serviços para o lazer e o aproveitamento da natureza (JUAREZ; CAÑETE, 2013).

Com isso, as intenções de turismo convertemse em oportunidade de captação monetária à medida que pessoas de origens diversas as visitam e consomem produtos e serviços (AGUIAR; CARVALHO, 2012, apud MESQUITA et al, 2015). Desta maneira, o turismo e a cultura se constituíram nas duas atividades mais rentáveis do século XX (RICHARDS, 2010, apud MESQUITA et al, 2015). A dimensão cultural do turismo envolve, portanto, a visita a locais históricos, sítios arqueológicos, museus e demais lugares que possibilitem experiências de conhecimento e participação na história do patrimônio visitado (FERREIRA, 2013, apud MESQUITA et al, 2015). As áreas de recreação oferecem uma série de benefícios, desde aqueles que podem ser valorados em mercados formais até os considerados intangíveis, que necessitam da aplicação de técnicas para estimar os seus valores. Assim, considerando as vantagens socioambientais que representam, os estudos de valoração econômica com vistas a quantificá-los são de extrema importância para fundamentar o processo de gestão das políticas públicas ambientais (SOUSA; MOTA, 2006).

É neste contexto que se insere a criação do Parque Nacional da Serra da Capivara em 1979, como uma forma de proteção aos mais de mil sítios arqueológicos encontrados na região (PESSIS; GUIDON, 2007, apud MESQUITA et al, 2015; ICMBIO, 2019) que juntos abrigam mais de 40.000 pinturas rupestres (OLIVEIRA FILHO; MONTEIRO, 2009, apud MESQUITA et al, 2015). Por ser bastante amplo geograficamente, o Parque se estende por quatro municípios: Brejo do Piauí, Coronel José Dias, João Costa e São Raimundo Nonato, todos no estado do Piauí, na região Nordeste do Brasil.

Diante da magnitude e da busca pela valoração, as análises econômicas do ambiente natural, que despontaram na década de 1960 e 1970 com intuito de promover o melhor aproveitamento dos recursos (ANGELO; 
CARVALHO, 2007) e de descobrir a importância que os indivíduos concedem às funções do meio ambiente, servirão de instrumento para alcançar o objetivo desta pesquisa. Deste modo, esta pesquisa procura estimar o valor de uso recreativo dos bens e serviços ecossistêmicos do Parque Nacional da Serra da Capivara por meio do um método de preferências reveladas, especificamente o Método de Custo de Viagem (MCV), através dos gastos incorridos pelos turistas que frequentam a região, a fim de investigar as variáveis explicativas acerca do perfil e da percepção do turista, e o que a experiência, produtos e serviços culturais têm a oferecer. A hipótese que norteia esta pesquisa é que a taxa e a frequência de visitas são influenciadas pelos custos de viagem e que a visitação diminui com o aumento da distância a percorrer.

\section{MATERIAIS E MÉTODOS}

\section{Área de estudo}

Criado pelo Decreto 83.548, de cinco de junho de 1979, com 129.139 hectares de área, o Parque Nacional Serra da Capivara é centro das atenções de arqueólogos e estudiosos do mundo inteiro (SETUR-PI, 2019). Localizado no semiárido nordestino, fronteira entre duas formações geológicas, com serras, vales e planície, o Parque abriga fauna e flora específicas da caatinga (ICMBIO, 2019). Ele possui uma variedade de objetos cadastrados e em estudo, os quais têm ajudado a esclarecer o povoamento das Américas, tais como urnas funerárias, sepultamentos indígenas, fósseis de mastodontes, cavalos, lhamas, tigres dente-de-sabre, preguiças e tatus gigantes e fósseis humanos, com datações que chegam há quatro mil anos e meio (SETUR-PI, 2019). As descobertas assumiram tamanha dimensão que em 1986 foi criada a Fundação Museu do Homem Americano - FUMDHAM, dirigida pela arqueóloga paulista Niède Guidon, responsável por buscar novos vestígios e catalogar descobertas, como manifestação da riqueza deixada pelas civilizações pré-históricas através de suas pinturas rupestres que representam rituais de caça, animais da época, cenas do cotidiano e figuras humanas (SETUR-PI, 2019).

De acordo com o Ministério do Turismo, o Parque tem capacidade para receber dois milhões de turistas por ano, dado que dispõe de infraestrutura com acesso a cento e setenta e três sítios arqueológicos abertos à visitação (MTUR, 2019), embora apresente uma média anual muito aquém disso, de apenas vinte mil visitas (MTUR, 2019). Quanto ao acesso, dispõe de trezentos quilômetros de trilhas carroçáveis e aproximadamente cem quilômetros sinalizados para pedestres, bem como adaptações para atendimento a pessoas com mobilidade reduzida (CARVALHO, 2012, apud MESQUITA et al, 2015). Em termos de recursos humanos, a pesquisa de referência indicou a disponibilidade de trinta e cinco condutores turísticos (OLIVEIRA FILHO; MONTEIRO, 2009, apud MESQUITA et al, 2015). Atualmente, não há cobrança de ingresso. $O$ serviço de guia (obrigatório nas atividades de circuito) é paga à parte aos condutores cadastrados (ICMBIO, 2019). Quanto às atividades econômicas desenvolvidas nos arredores, merece destaque a relevante presença do comércio de artesania, que retrata a cultura dos povos das cavernas e seu legado (OLIVEIRA FILHO; MONTEIRO, 2009, apud MESQUITA et al, 2015) e a agricultura de subsistência e criação de animais, já que a prática do turismo ganhou força na região somente após os anos de 1990 (MESQUITA et al, 2015).

Dotado de uma beleza extraordinária, o principal atrativo do Parque são as paisagens e os sítios arqueológicos com pinturas rupestres e grafismos gravados sobre os paredões areníticos (ICMBIO, 2019). Além da formação de cânions e gigantescas muralhas, ele preserva painéis que retratam a vida dos antigos habitantes, a fauna e a flora sertaneja. O complexo também inclui o Museu do Homem Americano que guarda um valioso acervo entre peças líticas, esqueletos, cerâmicas e artefatos de escavações arqueológicas, resultado de mais de quatro décadas de pesquisas feitas na região por universidades do Brasil e do mundo (MTUR, 2019). Em Coronel José Dias, encontra-se o Museu da Natureza, o qual oferece ao turista um passeio interativo mostrando a criação do universo, os impactos climáticos e as constantes transformações da fauna e da flora (MTUR, 2019).

O Boqueirão da Pedra Furada, monumento geológico símbolo da unidade e o maior painel natural de pinturas rupestres do planeta, guarda formas gráficas de comunicação da pré-história (MTUR, 2019). Além disso, o Parque reserva também sítios históricos, como casas de antigos maniçobeiros que habitaram o lugar, os quais viviam da coleta do insumo até meados do século XX, concentrados na região da Serra Branca - na Trilha dos Maniçobeiros. A fauna é variada com registro da presença de tatus-bola, tamanduás, jaguatiricas, jacus, cotias, veados-catingueiros, porcos-do mato, macacos-prego, onças, várias espécies de pássaros, lagartos e serpentes (ICMBIO, 2019).

Segundo estudo realizado sobre a Demanda Turística em 2012, o Piauí é o principal emissor de 
turistas para o Parque, respondendo na época por $56,3 \%$, seguido pelo Maranhão (17,3\%) (OSMAN; ASANO, 2012). Em análise anterior, Oliveira Filho e Monteiro (2009) descreveram a atratividade do sítio como ecológico, aventura, científico, voltado para a terceira idade e educacional, cuja visita inclui as seguintes atividades: 1) Visitar os sítios arqueológicos e históricos; 2) Caminhada; 3) Observar a paisagem nos mirantes; 4) Cicloturismo; 5) Observar os pássaros; 7) Visitar o Museu do Homem Americano; 8) Conhecer a produção de cerâmica; 9) Admirar a iluminação noturna; e 10) Conhecer o Centro de Visitantes que dispões de infraestrutura de apoio com banheiros, alimentação, auditório e loja de souvenirs, além de salão de exposição (ICMBIO, 2019).

\section{Método do Custo de Viagem}

De acordo com a teoria da maximização da utilidade, o problema de otimização apresentado no Método do Custo de Viagem (MCV) pode ser descrito, segundo Torres-Ortega et al (2018), da seguinte forma:

$$
\begin{gathered}
\text { Máx } U(q, I, z) \\
s a: C V * q+z=I
\end{gathered}
$$

Onde:

- U é a utilidade obtida do consumo de uma quantidade do bem;

- I é a renda;

- q é a quantidade consumida do bem;

- z corresponde ao consumo de outros bens;

- CV é o Custo da Viagem.

Assim, a demanda Marshalliana para um determinado destino se representa como:

$$
R=f(q, C V, z, I)
$$

O valor recreativo estimado pelo custo de viagem foi obtido por meio da multiplicação do somatório do excedente do consumidor de cada local de origem (ou zona) pelo número de visitas por ano, que corresponde a aproximadamente vinte mil turistas anuais.

Para a consecução do objetivo, a primeira etapa constituiu na aplicação questionários divididos em três blocos de questões: perfil socioeconômico (idade, nacionalidade, grau de instrução e renda mensal) e informações sobre o custo da viagem (origem, frequência de visitas, tempo de permanência, gastos para translado, alimentação, hospedagem e etc., e percepções sobre as atividades desenvolvidas durante o passeio). Em seguida, foram utilizadas análises descritivas para caracterizar o perfil dos visitantes.

Em relação à utilização do MVC, existem duas variantes clássicas (AZQUETA et al. 2007; ROBERT, 2017; VOLTAIRE et al., 2017), as quais podem ser o Método de Custo de Viagem Zonal (MCVZ), cujo objetivo é estimar a propensão média a visitar um determinado destino desde as diferentes zonas em que sua área de influência está dividida, ou o Método de Custo de Viagem Individual (MCVI), que busca descobrir a demanda individual de serviços de lazer em um destino. A principal diferença é que uma utiliza dados individuais em vez de zonais, revelados a partir de pesquisas aplicadas a seus visitantes. No presente estudo, optou-se pela utilização do MCVZ.

\section{Procedimento do $M C V Z$}

A aplicação do método de custo de viagem se deu em cinco fases:

Fase 1: Determinou-se o número de visitantes (Vi) e se realizou análise da frequência de visitas ao Parque mediante questionário distribuído online.

Fase 2: Foi calculada a taxa de visitas ao Parque (TVi). A fórmula corresponde a taxa de visitas por mil pessoas ao ano (ANGELO; CARVALHO, 2007).

$$
\frac{T V_{i}}{1000}=\frac{\left[\left(\frac{V_{i}}{n}\right) * N * 1000\right]}{P_{i}}
$$

Onde:

- $\mathrm{V}_{\mathrm{i}}$ : é o número de visitantes da zona i;

- n é o tamanho da amostra (número total de entrevistados);

- N é o número total de visitantes por ano;

- Pi é a população total da zona i.

Fase 3: Determinação do Custo de Viagem Total $\left(\mathrm{CV}_{\mathrm{i}}\right)$, medido a partir da soma do custo de deslocamento (gastos com combustível e/ou passagens para chegar até o local de destino) e do custo de permanência (despesas de hospedagem, alimentação, entrada no Parque e outros gastos extras), dos visitantes. Não foi avaliado o custo de oportunidade do tempo, pois, como afirma Tahzeeda, Khan e Bashar (2018), sendo a maioria dos visitantes estudantes, assume-se que no seu tempo de ócio eles se encontram em momentos ou trabalhos não monetários e que outras pessoas também visitam a área durante as tardes ou nas férias. Outros autores que incluem o valor do tempo, como Shammin (1999), Freeman, Herriges e Kling (2014), utilizam o valor de um terço do salário regular, ou um terço do salário por hora 
(PARSONS, 2013). Assim, é possível afirmar que a inclusão do valor do tempo envolve o julgamento próprio do consumidor (SIMÕES, BARATA e CRUZ, 2013).

Fase 4: Obtenção da curva de demanda de visitas para as atividades recreativas no Parque segundo cada região, utilizando o valor de custo total médio de viagem para verificar a variação da taxa de visitas quando se altera aumenta o dispêndio médio.

Fase 5: Estimou-se o Valor de Uso Recreativo (VUR) do Parque, retratado pela área sob a curva que representa o Excedente do Consumidor (EC) e reflete o valor de uso recreativo baseado na análise do método de custo de viagem a partir da fórmula:

$$
\begin{aligned}
& V U R_{\text {ESTIMADO ANUAL }}=E C_{P / \text { VISITANTE }} * N \\
& E C_{P / \text { VISITANTE }}=\frac{E C_{\text {TOTAL }}}{n} \\
& E C_{\text {TOTAL }}=\int_{C V_{i_{\text {min }}}}^{C V_{i_{\text {máx }}}} f(x) d x-\left(C V_{i_{\text {máx }}}-C V_{i_{\text {min }}}\right)
\end{aligned}
$$

Onde:

- CVimín: Custo da Viagem mínimo entre as zonas observadas;

- CVimáx: Custo da Viagem máximo entre as zonas observadas;

- f(x): Função de demanda das visitas ao Parque;

- TVimín: Taxa de visitas mínima desde as i zonas observadas até o Parque.

O Excedente do Consumidor (EC) é a diferença entre a quantidade total que os consumidores estão dispostos a pagar por um bem ou serviço e o valor total que eles realmente pagam, compreendido como uma medida do benefício líquido dos visitantes (TAHZEEDA, KHAN e BASHAR, 2018; TORRES-ORTEGA et al, 2018).

Além do Valor de Uso Recreativo Estimado Anual, resultou interessante estimar o Valor de Uso Recreativo Potencial Anual da seguinte forma:

$$
V U R_{P O T E N C I A L A N U A L}=E C_{P / V I S I T A N T E} * \sum_{i=1}^{z_{i}} \frac{P_{i}}{g_{i}}
$$

Onde:

- $\quad P_{i}$ : População da zona i que visitou o Parque;

- $\quad g_{i}$ : Grupos familiares ou média de moradores em domicílios particulares permanentes da zona i que visitaram o Parque;

- $\quad z_{i}$ : Zona i desde onde tem se registrado visitas ao Parque.

\section{Dados e amostragem}

A pesquisa foi realizada através de uma amostragem aleatória simples para a obtenção do tamanho de amostra ótimo, conforme a expressão de Parga e Alonso (2018), com um nível de confiança do $95 \%, \mathrm{p}=0,5$ e um erro do $10 \%$. Obtiveram-se noventa e oito respostas, delas 81 válidas e 17 consideradas pilotos para testar a plataforma e avaliar as questões.

$$
n=\frac{z^{2} * N * p * p}{N * E+z^{2} * p * p}
$$

Onde:

- N é o tamanho da população;

- $z^{2}$ é o valor crítico para 1- $\alpha$ (nível de confiança);

- $\mathrm{p}=\mathrm{q}=0,5$ é a máxima variabilidade da proporção;

- E é o erro máximo permissível

De acordo com os dados fornecidos pelo Parque, são registrados um total de 20.000 visitantes por ano, que representa uma média de 384 visitantes por semana. Dessa forma, obtém-se um tamanho de amostra ótimo de 77 respondentes sendo que foram considerados válidos 81 questionários. É interessante mencionar que o erro do $10 \%$ pode se justificar por razões de tempo, disponibilidade de recursos, logística, deslocamento até o local, tal como em Barros et al, (2018), Tyskowski et al, (2018) e Lima et al, (2019).

O instrumento foi elaborado com 31 questões que traçaram o perfil sociodemográfico dos visitantes, assim como descreveu os custos de viagem, o nível de satisfação para com as atrações disponíveis e a percepção sobre a qualidade e acesso ao local. Todos os respondentes foram voluntários na pesquisa e não houve qualquer identificação pessoal de suas respostas. $\mathrm{Na}$ sequência, o questionário foi distribuído aleatoriamente durante o mês de junho de 2019 por meio de plataforma online, tendo sido compartilhado em diversas páginas de turismo, de universidades e de rádios locais, buscando-se a obter a maior amostra possível e ao mesmo tempo capturar a percepção da população local. Para reduzir o risco de duplicidade, foi adotada restrição de envio de uma resposta por IP.

Para responder as perguntas, aplicou-se o método de valoração ambiental de Custo da Viagem, sendo necessário os visitantes haver estado presencialmente pelo menos uma vez no local, contextualizando-o como um destino turístico. Foram entrevistados turistas brasileiros, homens e mulheres, com idades 
variadas e origem em catorze cidades distintas, de cinco unidades federativas e de dois países, de vinte e cinco ocupações profissionais, com níveis de renda diversos e que utilizaram até três tipos de modais para acessar o Parque.

\section{RESULTADOS E DISCUSSÕES}

\section{Perfil dos visitantes}

Do total de noventa e oito respondentes, oitenta e uma pessoas afirmaram ter visitado o Parque Nacional da Serra da Capivara, sendo que aproximadamente $\quad 45,7 \%$ indicaram haver visitado pelo menos uma vez no ano, $21,0 \%$ já visitou mais de três vezes, $23,5 \%$ foi duas vezes e $9,9 \%$, três vezes. No que diz respeito à diferenciação sexual, predominou visitantes do sexo feminino e idade de 18 a 35 anos (ambos $60,5 \%$ ), e grau de escolaridade superior completa $(81,4 \%)$, sendo que $14,8 \%$ indicou graduação incompleta e $2,5 \%$, ensino médio incompleto. $\mathrm{O}$ nível de renda mensal dos turistas apontou que $50,6 \%$ possui recebimentos maiores que cinco salários mínimos e $21 \%$ recebe de um a três salários mínimos mensalmente, reforçando a hipótese de que o custo de viagem influencia na demanda de visitantes, prevalecendo o perfil socioeconômico pertencente a faixas de renda mais altas. É válido recordar que, no período em que as entrevistas foram aplicadas, o valor do salário mínimo brasileiro era de $\mathrm{R} \$ 998,00$ (BACEN, 2019). As classificações sociodemográficas utilizadas foram baseadas nos indicadores sociais do IBGE (2019). A tabela 1 resume os resultados sociodemográficos coletados.

Tabela 1. Dados Sociodemográficos

\begin{tabular}{llcc}
\hline Variável & & $\mathbf{n}$ & $\mathbf{\%}$ \\
\hline Gênero & Feminino & 49 & 60,5 \\
& Masculino & 32 & 39,5 \\
\hline Faixa etária & Menos de 18 anos & 2 & 2,5 \\
& 18 a 35 anos & 49 & 60,5 \\
& 36 a 59 anos & 19 & 23,5 \\
& Tenho 60 anos ou mais & 10 & 12,3 \\
\hline Grau de escolaridade & Ensino médio incompleto & 2 & 2,5 \\
& Graduação completa & 15 & 18,5 \\
& Graduação incompleta & 12 & 14,8 \\
& Especialização incompleta & 7 & 8,6 \\
& Especialização completa & 23 & 28,4 \\
& Mestrado incompleto & 3 & 3,7 \\
& Mestre & 9 & 11,1 \\
& Doutorado incompleto & 1 & 1,2 \\
& Doutor(a) & 8 & 9,9 \\
\hline Nível de renda per capita & até 1 salário mínimo & 5 & 6,2 \\
& de 1 a 3 salários mínimos & 17 & 21,0 \\
& de 3 a 5 salários mínimos & 18 & 22,2 \\
& mais de 5 salários mínimos & 41 & 50,6 \\
\hline
\end{tabular}

Fonte: Elaborada pelos autores (2020).

Em relação às ocupações declaradas, é destaque que $29,6 \%$ é composto por professores e por estudantes, $12,3 \%$ por médicos, $3,7 \%$ por arqueólogos e 2,5\% por aposentados. Após montar um perfil socioeconômico dos respondentes, as interrogações deram seguimento a busca sobre informações referentes à viagem em si.
Foram consideradas as entrevistas com turistas provenientes de cinco Estados brasileiros (Distrito Federal, Maranhão, Minas Gerais, Piauí e São Paulo), sendo 71,6\% oriundos de Teresina e $7,4 \%$ de São Raimundo Nonato, ambas no Piauí, percorrendo em média 632,93 quilômetros. 
Tabela 2. Cidades de origem dos respondentes

\begin{tabular}{lcc}
\hline Origem & N & $\mathbf{\%}$ \\
\hline Belo Horizonte-MG & 1 & 1,2 \\
Bonfim do Piauí-PI & 2 & 2,5 \\
Brasília-DF & 2 & 2,5 \\
Buriti dos Lopes-PI & 1 & 1,2 \\
Canto do Buriti-PI & 1 & 1,2 \\
Floriano-PI & 1 & 1,2 \\
Cayenne/Guiana Francesa & 1 & 1,2 \\
Parnaíba-PI & 2 & 2,5 \\
Santos-SP & 1 & 1,2 \\
São Paulo-SP & 1 & 1,2 \\
São Raimundo Nonato-PI & 6 & 7,4 \\
Teresina-PI & 58 & 71,6 \\
Timon-MA & 1 & 1,2 \\
Varginha-MG & 1 & 1,2 \\
Várzea Branca-PI & 2 & 2,5 \\
\hline
\end{tabular}

Fonte: Elaborada pelos autores (2020).

Com relação ao tipo de meio de transporte utilizado para chegar até o Parque, em geral, a viagem foi feita em veículo particular e de ônibus. $59,3 \%$ das pessoas indicaram uso de carro particular, $34,6 \%$ apontou o ônibus como meio de locomoção e $3,7 \%$ utilizou avião para acessar o sítio turístico. Para estimar os gastos com deslocamento via automóvel e criar um parâmetro de comparação com os valores declarados nas respostas, também foi registrado o tipo de carro utilizado pelas quarenta e oito pessoas que indicaram o modelo e calculada a estimativa de gasto com deslocamento médio por quilômetro por cada tipo, com base em informações do INMETRO (2019).

Tabela 3. Tipo de veículo utilizado para acessar

\begin{tabular}{lcc}
\multicolumn{3}{c}{ o Parque } \\
\hline Tipo de Carro & N & \% \\
\hline 1.0 & 8 & 16,7 \\
1.6 & 18 & 37,5 \\
2.0 & 14 & 29,2 \\
Mais de 2.0 & 8 & 16,7 \\
\hline
\end{tabular}

Fonte: Elaborada pelos autores (2020).

Os principais objetivos ao realizar o passeio turístico, conforme explicitado pelos respondentes, indicam que $35,8 \%$ das pessoas foram para o Parque pois estavam de férias e o mesmo percentual foi passar o fim de semana, $14,8 \%$ estava a trabalho e aproveitou a oportunidade de estar ali para conhecer o Parque, e 3,7\% apenas estava de passagem pela cidade e resolveu visitar o sítio. É resultante também que $77,8 \%$ dos visitantes ficou pelo menos dois dias na cidade e que $45,7 \%$ deles foram em grupos de quatro ou mais pessoas. Além disso, também foi consultada a quantidade de horas que as pessoas ficaram no Parque, cuja 44,4\% das respostas apontou permanência de três a seis horas no local.

Tabela 4. Duração do passeio

\begin{tabular}{|c|c|c|}
\hline $\begin{array}{l}\text { Tempo de Permanência nc } \\
\text { Parque }\end{array}$ & $\mathbf{n}$ & $\%$ \\
\hline Até 3 horas & 10 & 12,3 \\
\hline Entre 3 e 6 horas & 36 & 44,4 \\
\hline Entre 6 e 8 horas & 17 & 21,0 \\
\hline Entre 8 e 12 horas & 6 & 7,4 \\
\hline Mais de 12 horas & 12 & 14,8 \\
\hline
\end{tabular}

Fonte: Elaborada pelos autores (2020).

$\mathrm{Na}$ sequência, o questionário avaliou a percepção dos visitantes sobre as atrações oferecidas, a partir da qualificação em escalas de 1 a 7 para cada uma. A satisfação da atividade turística identifica especialmente na beleza natural, cuja melhor avaliação foi para observar a paisagem nos mirantes com média 6,46 , seguida por visitar o Museu da Natureza com 6,33 e visitar os sítios arqueológicos com 6,25. Cabe ressaltar que nem todo mundo respondeu todos os itens, demonstrando não ter conhecido tal atividade, a exemplo de cicloturismo (índice de resposta de apenas 51,9\%) e de admirar a iluminação noturna (índice de $65,4 \%$ de resposta).

\section{Aplicação do Método do Custo de Viagem Zonal (MCVZ)}

A partir da aplicação do método, abaixo resumemse as informações sobre o número de visitantes, a taxa de visitas ao Parque e o custo de viagem total apresentados na tabela 5 . 
Tabela 5. Estimação das taxas de visitas e os custos totais da viagem

\begin{tabular}{lccccc}
\hline Cidade de origem & $\begin{array}{c}\text { Distância* } \\
\text { (ida e volta) }\end{array}$ & População & $\begin{array}{c}\text { Visitantes } \\
\text { (Obs.) }\end{array}$ & TV $_{\mathbf{i}}^{* * *}$ & CV $_{\mathbf{i}}^{* * * *}$ \\
\hline Bonfim do Piauí-PI & 126,2 & 5670 & 2 & 87,09 & 150 \\
Canto do Buriti-PI & 152,6 & 21187 & 1 & 11,65 & 145 \\
Floriano-PI & 438 & 59935 & 1 & 4,12 & 660 \\
São Raimundo Nonato-PI & 73,8 & 34710 & 6 & 42,68 & 192,8 \\
Teresina-PI & 812 & 864845 & 58 & 16,56 & 816,04 \\
Timon-MA & 800 & 169107 & 1 & 1,46 & 550 \\
Várzea Branca-PI & 146,6 & 4947 & 2 & 99,82 & 115 \\
Buriti dos Lopes-PI & 1236 & 19781 & 1 & 12,48 & 950 \\
Parnaíba-PI & 1300 & 153078 & 2 & 3,23 & 1010 \\
Belo Horizonte-MG & 2510 & 2512070 & 1 & 0,10 & 1470 \\
Brasília-DF & 1962 & 3015268 & 2 & 0,16 & 1228,5 \\
Santos-SP & 3482 & 433311 & 1 & 0,57 & 2550 \\
São Paulo-SP & 3412 & 12252023 & 1 & 0,02 & 3100 \\
Varginha-MG & 2922 & 135558 & 1 & 1,82 & 2100 \\
Cayenne/Guiana Francesa & 3716 & 57615 & 1 & 4,29 & 3000 \\
\hline
\end{tabular}

Fonte: Elaborada pelos autores (2020). * Distância estimada $(\mathrm{km})$ da viagem de ida e volta até o destino. ** Taxa de visitas de cada zona ou cidade de origem por cada 1000 pessoas. *** O valor do custo da viagem está estimado em $\mathrm{R} \$$ e representa os custos totais de deslocamento e permanência dos visitantes de cada zona ou cidade de origem.

Do exercício para a obtenção da curva de demanda de visitas para as atividades recreativas resultou a Figura 1, que relacionada a taxa de visitação a cada mil pessoas e o custo de viagem total a partir da equação seguinte equação, com
$\mathrm{R}^{2}=0,788, \mathrm{R}^{2}$ ajustado $=0,773, \mathrm{~F}-\mathrm{Fisher}=51,975$ e valor-p $=0,000$.

$$
T V_{i}=8754,868 * C V_{i}^{-1}
$$

Figura 1. Curva da demanda de visitas por cidade de origem

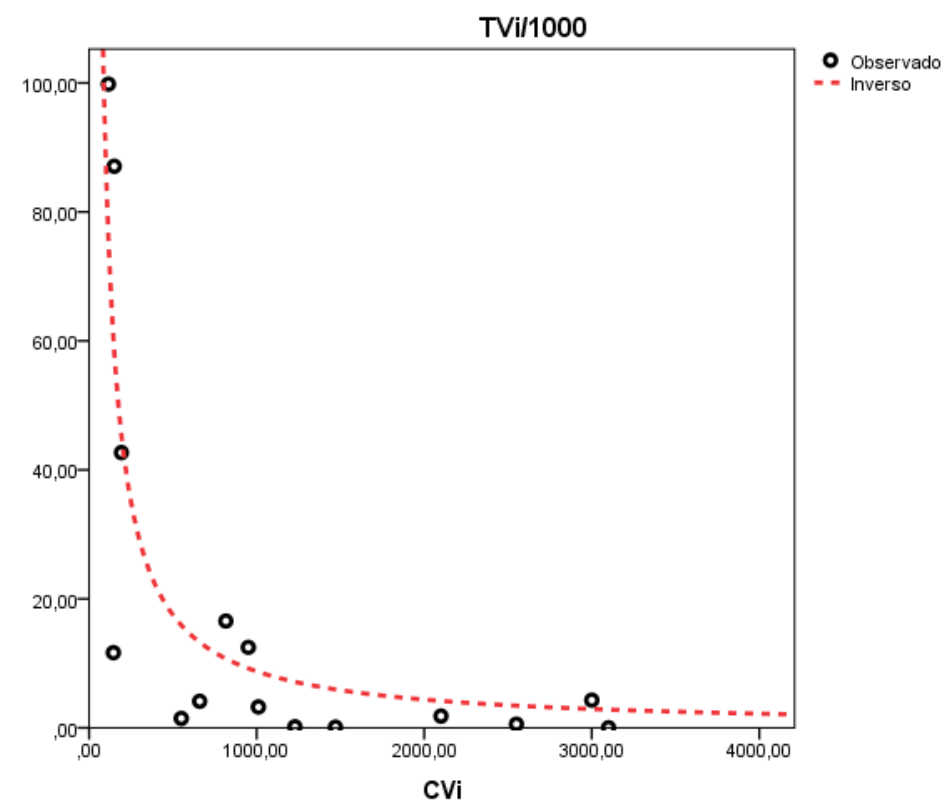

Fonte: Elaborado pelos autores (2020).

Demonstra-se, portanto, que os custos da viagem influenciaram a taxa de visita por mil pessoas, por isso Teresina soma mais de setenta por cento, enquanto cidades mais distantes possuem taxa de visitação menor. E por fim, sobre a estimação do Valor de Uso Recreativo do Parque, foram alcançados os seguintes resultados: 


$$
\begin{gathered}
E C_{\text {TOTAL }}=\int_{C V_{i_{\text {min }}}}^{C V_{i_{\text {máx }}}}\left(8754,868 * C V_{i}^{-1}\right) d C V_{i} \\
-\left(C V_{i_{\text {máx }}}-C V_{i_{\min }}\right) * T V_{i_{\text {min }}} \\
E C_{\text {TOTAL }}=8754,868 *\left(\left.\left(\ln C V_{i}\right)\right|_{115} ^{3100}\right) \\
-\left(C V_{\left.i_{\text {máx }}-C V_{i_{\text {min }}}\right) * T V_{i_{\text {min }}}}\right. \\
E C_{\text {TOTAL }}=R \$ 28780,81 \\
E C_{P / V I S I T A N T E}=R \$ 355,32 \\
V U R_{\text {ESTIMADO ANUAL }}=R \$ 7106372,18 \\
V U R_{\text {POTENCIAL ANUAL }}=R \$ 2199082256,11
\end{gathered}
$$

Ao analisar o excedente do consumidor de cada uma das origens, ou seja, o benefício final estimado, o qual foi calculado para obter o valor econômico agregado pela recreação ao Parque Nacional da Serra da Capivara, foi encontrado o total de $\mathrm{R} \$ 7,1$ milhões por ano, que representa a soma do excedente do consumidor das regiões registradas na pesquisa, multiplicado pelo total de visitantes anual. Cabe mencionar que possivelmente este valor esteja subestimado, visto que utiliza a estimativa mínima do número de turistas que visitam a região anualmente (20.000) e desconsidera o custo de oportunidade do tempo. Considerando esta possibilidade, foi calculado o valor de uso recreativo potencial e obtido o total de $\mathrm{R} \$ 2,2$ bilhões.

Paralelamente, optou-se por desenvolver a análise do custo de viagem a partir dos modelos de demanda de Fasciolo (2002) de aplicação do Método de Custo de Viagem Zonal, considerando os respondentes válidos no total de 81 , a título de realizar a comparação dos resultados, utilizando um modelo de regressão simples que propõe a TVi como uma variável dependente explicada, no sentido geral, pelo Custo Total da Viagem, cujos modelos estimados podem ser vistos na tabela 6 . Continua sendo custo da viagem zonal porque as TVi são das cidades de origem de cada respondente, e não são analisadas as características socioeconômicas dos indivíduos, sendo reconhecida na literatura também como uma forma de MCVZ.

Tabela 6. Modelos estimados para o Método de Custo de Viagem Zonal $\left(\mathrm{TV}_{\mathrm{i}}\right)$

\begin{tabular}{lcccccc}
\hline Variável & Linear & Logarítmico & Inverso & Potência & Exp & Logístico \\
\hline \multirow{2}{*}{ Constante } & $31,889^{* *}$ & $124,777^{* *}$ & $5,403^{* *}$ & $6869,995^{* *}$ & $36,777^{* *}$ & $0,027^{* *}$ \\
& $(0,000)$ & $(0,000)$ & $(0,000)$ & $(0,000)$ & $(0,000)$ & $(0,000)$ \\
\hline \multirow{2}{*}{$\mathrm{CV}_{\mathrm{i}}$} & $-0,014^{* *}$ & $-16,237^{* *}$ & $6552,749^{* *}$ & $-0,980$ & $-0,001^{* *}$ & $1,001^{* *}$ \\
\hline $\mathrm{R}^{2}$ & $(0,000)$ & $(0,000)$ & $(0,005)$ & $(0,345)$ & $(0,000)$ & $(0,000)$ \\
\hline $\mathrm{R}^{2}$ ajustado & 0,214 & 0,464 & $\mathbf{0 , 6 1 0}$ & 0,317 & 0,344 & 0,344 \\
\hline F-Fisher & 0,204 & 0,457 & $\mathbf{0 , 6 0 5}$ & 0,308 & 0,336 & 0,336 \\
\hline $\mathrm{df} 1$ & 21,558 & 68,258 & 123,427 & 36,606 & 41,397 & 41,397 \\
\hline df2 & 1 & 1 & 1 & 1 & 1 & 1 \\
\hline $\mathrm{p}$-valor & 79 & 79 & 79 & 79 & 79 & 79 \\
\hline
\end{tabular}

Fonte: Elaborada pelos autores (2020). * Significativo $\mathrm{p}<0,05$ (95\% de confiança) ${ }^{* *}$ Significativo $\mathrm{p}<$ $0,01(99 \%$ de confiança)

Entre os modelos testados, o que apresentou melhor ajuste foi o inverso, explicando mais de sessenta por cento da taxa de visitação do Parque. A partir da escolha, foi formada a seguinte equação para determinar a $\mathrm{TV}_{\mathrm{i}}$ :

$$
T V_{i}=5,403+6252,749 * C V_{i}^{-1}
$$

Aplicou-se na fórmula do excedente do consumidor e alcançou-se os resultados a seguir:

$$
\begin{gathered}
E C_{\text {TOTAL }}=\left.\left(5,403 * C V_{i}+6252,749 * \ln C V_{i}\right)\right|_{82} ^{3100} \\
-\left(C V_{i_{\text {máx }}}-C V_{i_{\min }}\right) * T V_{i_{\text {min }}} \\
E C_{\text {TOTAL }}=R \$ 38958,62 \\
E C_{P / V I S I T A N T E}=R \$ 480,97 \\
V U R_{\text {ESTIMADO ANUAL }}=R \$ 9619411,84 \\
V U R_{\text {POTENCIAL ANUAL }}=R \$ 2976747820,40
\end{gathered}
$$

Da mesma maneira, a Figura 2 apresenta a estimação da respectiva curva de demanda de visitas de acordo com o modelo definido por Fasciolo (2002).

Para esta análise, o valor de uso recreativo estimado para o Parque Nacional da Serra da Capivara encontrado foi de $\mathrm{R} \$ 9,6$ milhões por ano. E o valor de uso recreativo potencial obtido foi de $\mathrm{R} \$ 3$ bilhões aproximadamente.

\section{CONCLUSÕES}

Uma vez alcançado o objetivo deste artigo, o qual foi designado a estimar o valor de uso recreativo dos bens e serviços ecossistêmicos do Parque Nacional da Serra da Capivara por meio de um método de preferências reveladas, especificamente o Método de Custo da Viagem, 
confirmou-se o pressuposto de que a frequência de visitas é influenciada pelos custos de viagem. Mesmo com provável subestimação decorrente da imprecisão de registros do número de turistas que frequentam o local, tem-se que o valor de uso recreativo do Parque é de aproximadamente $\mathrm{R} \$ 7,1$ milhões por ano, correspondendo a cerca de 0,2\% do valor do PIB do Estado de Piauí em 2017 - último valor disponível de $\mathrm{R} \$ 45,4$ bilhões, dependendo do modelo usado para estimar o VUR e um potencial de representar até $6,6 \%$ do PIB estadual, sendo seguramente um indicativo da importância da preservação do patrimônio natural.

Neste sentido, como uma forma de contribuição para o Parque, poderia se avaliar a captação de recursos dos visitantes a partir da sua disponibilidade a pagar pelos serviços ecossistêmicos, sem que isto represente de modo algum uma privatização do ativo ambiental ou fixação de preço de mercado, e sim uma percepção de valorização do espaço. A partir de uma concepção econômica, esta valorização estaria representada pelo desejo total a pagar pelos usuários do Parque, podendo assim ser revertido para fins estritamente de proteção e conservação do espaço natural.

É senso comum para muitos autores que desenvolver estratégias marketing e programas de divulgação e educação científicas, bem como ações práticas e eficientes de educação ambiental direcionadas a este tipo de ecossistema seriam indicadas para fomentar o conhecimento dos turistas e não turistas sobre o local e auxiliar na percepção do valor dos bens e serviços disponíveis e sobre a importância da atitude individual na manutenção destes recursos.

Figura 2. Curva de demanda de visitas segundo modelo de Fasciolo (2002)

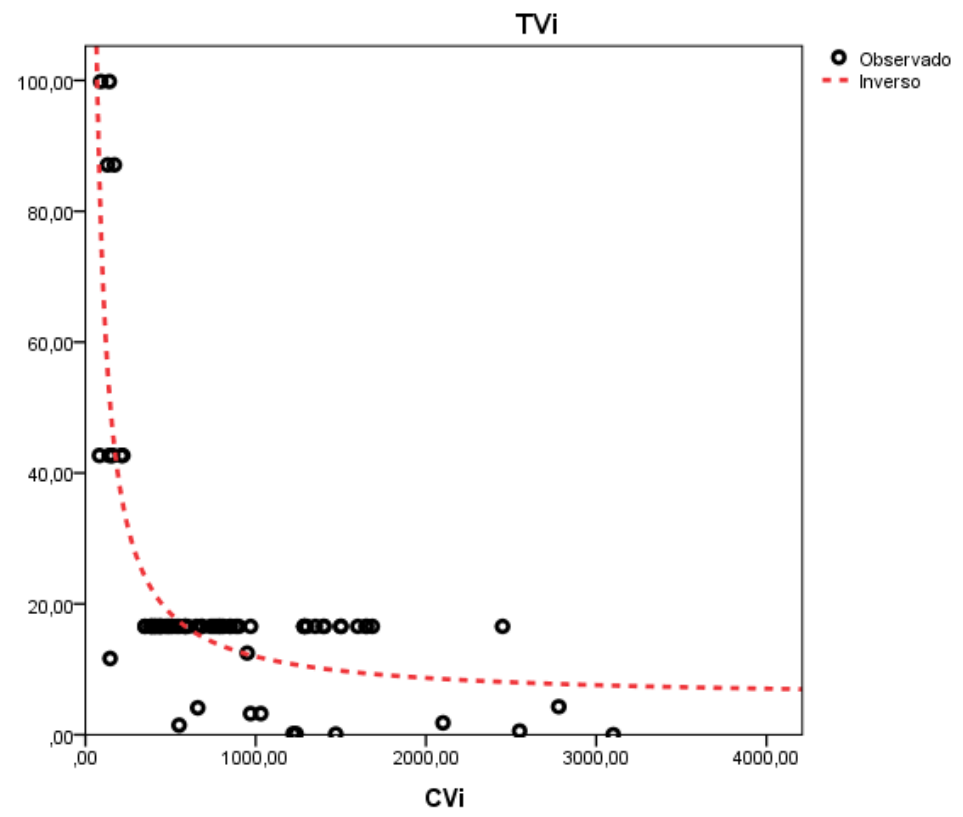

Fonte: Elaborado pelos autores (2020).

Para futuros estudos, sugere-se aprofundar a análise do comportamento dos usuários, sabendo que a cidade de São Raimundo Nonato possui acesso a práticas de ecoturismo - em processo de intensificação - que não foram evidenciadas neste estudo, abrindo espaço para investigação desta dimensão sustentável das atividades turísticas.

Assim, diante da grandeza e dos atributos do Parque Nacional da Serra da Capivara, e levando em consideração que o turismo presente é uma atividade baseada tanto na cultura como na história e na natureza, é condição que o lugar e seus bens e serviços sejam destino de investimentos ambientais que assegurem a manutenção da qualidade do capital natural e o bem-estar proporcionado aos visitantes, bem como a continuidade de seu uso e o benefício econômico que este ambiente gera.

\section{REFERÊNCIAS}

ANGELO, P. G., CARVALHO, A. R. Valor recreativo do rio Araguaia, região de Aruanã, estimado pelo método do custo de viagem. Acta Sci. Biol. Sci Maringá, v. 29, n. 4, p. 421-428, 2007. https://doi.org/10.4025/actascibiolsci.v29i4.886

AZQUETA, D., ALVIAR, M., DOMINGUÉZ, L., O'RYAN, R. Introducción a la Economía Ambiental. 2 ed., Madrid: McGrawHill/Interamericana de España, S.A.U, 2007. 
BACEN. Banco Central do Brasil. Sistema Gerenciador de Séries Temporais v2.1. Brasília, 2019. Disponível em: <https://www3.bcb.gov.br/sgspub/consultarvalor es/consultarValoresSeries.do? method=getPagina >. Acessado em 04/08/2019.

BARROS, D. CASTRO, R. V. O, CARNEIRO, A. de C. O., CARVALHO, A. M. M. L, VITAL, B. R., SANTOS, R. C. dos. Sampling methods and number of roundwoods for determining the moisture content. Revista Árvore, v. 42, n. 4: e420401, p.1-6, 2018 . http://dx.doi.org/10.1590/180690882018000400001

IBGE. Instituto Brasileiro de Geografia e Estatística. Síntese de Indicadores SociaisSIS. 2019. Disponível em: <https://www.ibge.gov.br/estatisticas/sociais/pop ulacao/9221-sintese-de-indicadoressociais.html?edicao $=9222 \& \mathrm{t}=$ sobre $>$. Acessado em 04/08/2019.

ICMBIO. Instituto Chico Mendes de Conservação da Biodiversidade. Parque Nacional da Serra da Capivara. 2019. Disponível em: <http://www.icmbio.gov.br/portal/visitacao1/unid ades-abertas-a-visitacao/199-parque-nacionalda-serra-da-capivara>. Acessado em 27/07/2019.

INMETRO. Instituto Nacional de Metrologia, Qualidade e Tecnologia. Tabelas de Consumo/Eficiência Energética: Veículos Automotores Leves. 2019. Disponível em: <http://www.inmetro.gov.br/consumidor/pbe/veic ulos_leves_2019.pdf>. Acessado em 26/07/2019.

FASCIOLO, G. Método de Valoración Contingente $(M V C)$. Instituto Nacional del Agua. Centro de Economía, Legislación y Administración del Agua, 2002.

FUMDHAM. Fundação Museu do Homem Americano. Parque Nacional da Serra da Capivara. 2019. Disponível em: <http://fumdham.org.br/> Acessado em 27/07/2019.

FREEMAN, A. M., III, J. A. HERRIGES e C. L. KLING. The Measurement of Environmental and Resource Values: Theory and Methods. Routledge. 3rd Edition., New York: Resource for the Future (RFF) Press, 2014.

JUAREZ, A. S.; CAÑETE, R. B. Valuation of the recreational use of the Calares del Mundo and Sima Natural Park through the travel cost method. Instituto Nacional de Investigación y Tecnología Agraria y Alimentaria (INIA), Forest Systems, v.22, n. 2, p.189-201, 2013. https://doi.org/10.5424/fs/2013222-02534

LIMA, M. MIRANDA, L da S. BARROS, W dos S., COSTA, B. C., OLIVEIRA, E. S. de, RADDATZ, D. D., OLIVEIRA, F. de A. Comparação entre processos de amostragem para estimar o volume em uma florestal no município de Barcarena-PA. Meio Ambiente: Inovação com Sustentabilidade. Ponta Grossa: Editora Antena, v.1, p. 158-167, 2019. https://doi.org/10.22533/at.ed.454190110

MESQUITA, R. F. de, SOUSA, L. R. M., MATOS, F. R. N., MONTE, A. L. A. do. Comportamento turístico dos visitantes do Parque Nacional da Serra da Capivara. Tourism \& Management Studies, v.11, n. 2, 2015. p. 78-85, 2015. https://doi.org/10.18089/tms.2015.11210

MTUR. Ministério do Turismo. Ministro do Turismo interino faz visita técnica à Serra da Capivara (PI). 2019. Disponível em: $<$ http://www.turismo.gov.br/\%C3\%BAltimasnot\%C3\%ADcias/12819-ministro-do-turismointerino-faz-visita-t\%C3\%A9cnica-\%C3\%A0serra-da-capivara-pi.html>. Acessado em 27/07/2019.

OLIVEIRA FILHO, R. C. de, MONTEIRO, M. do S. L. Ecoturismo no Parque Nacional Serra da Capivara: trata-se de uma prática sustentável? Turismo em Análise, v. 20, n. 2, Teresina, 2009.

OSMAN, A., ASANO, L. Trecho turístico do Parque Nacional da Serra da Capivara: entraves, análise e propostas de melhoria. Fundação Getúlio Vargas, São Paulo, 2017.

PARGA, E.; ALONSO, P. The Altamira controversy: Assessing the economic impact of a world heritage site for planning and tourism management. Journal of Cultural Heritage, v. 30, p. 180-189, 2018. https://doi.org/10.1016/j.culher.2017.09.007

PARSONS, G. R. The Travel Cost Model. In A Primer on Nonmarket Valuation. The Economics of Non-Market Goods and Resources. Dordrecht: Kluwer Academic Publishers, 2013. p. 269329.

ROBERT, I. Application of Travel Cost Method to Valuation of Historic Building: Old Residence in Calabar, Nigeria: Middle-East Journal of Scientific Research, v. 25, n. 10, 2017. p. 1925933.

https://doi.org/10.5829/idosi.mejsr.2017.1925.19 33

SETUR-PI. Secretaria de Estado de Turismo do Piauí. Governo do Estado do Piauí. Parque Nacional da Serra da Capivara. 2019. Disponível em: <http://www.turismo.pi.gov.br/serracapivara/>. Acessado em 27/07/2019.

SHAMMIN. Application of the travel cost method (TCM): A case Study of Environmental valuation of Dhaka Zoological Garden. IUCN: Switzerland, 1999.

SIMÕES, P., BARATA E. e CRUZ, L. Joint Estimation Using Revealed and Stated Preference Data: $\quad$ An Application Using a National Forest. Journal of Forest Economics, n. 19, p. $249-$ 266 ,

2013. https://doi.org/10.1016/j.jfe.2013.03.001

SOUSA, G. B. de; MOTA, J. A. Valoração econômica de áreas de recreação: o caso do Parque Metropolitano de Pituaçu, Salvador: Revista de Economia, Editora UFPR, v. 32, n. 1, ano 30, p. 37-55, 2006. 
TAHZEEDA, J., KHAN, M.R., BASHAR, R. Valuation approaches to ecosystem goods and services for the National Botanical Garden. Bangladesh: Environmental \& Socioeconomic Studies, n. 6(1), p. 1-9, 2018. https://doi.org/10.2478/environ-2018-0001

TORRES-ORTEGA, S., PÉREZ-ÁLVAREZ, R., DÍAZ-SIMAL, P., LUIS-RUIZ, J. M. de, PIÑAGARCÍA, F. Economic Valuation of Cultural Heritage: Application of Travel Cost Method to the National Museum and Research Center of Altamira. Sustainability, n. 10, p. 2550-2562, 2018. https://doi.org/10.3390/su10072550

TYSKOWSKI, K.C., AMARAL, J. A. da S., SOUSA, K. A., SANTOYO, A. H, PIACENTI, C. A. Estimación del valor de uso recreativo del Parque Ambiental "Horto Florestal" en Rio Branco, Acre. Revista Brasileira Planejamento e Desenvolvimento, v. 7, n. 5, Edição Especial Desenvolvimento Sustentável Brasil/Cuba, 2018. https://doi.org/10.3895/rbpd.v7n5.9059

VOLTAIRE, L. LÉVI, L., ALBAN, F., BONCOEUR, J. Valuing cultural world heritage sites: an application of the travel cost method to MontSaint-Michel. Applied Economics, v. 49 (16), p. 1593-1605, 2017.

https://doi.org/10.1080/00036846.2016.1221046 